Reprod. Nutr. Dévelop., 1982, 22 (1 B), 163-170.

\title{
Le néo-cortex chez l'embryon et le fœetus humain. Apport du microscope électronique et du Golgi
}

\author{
Jeanne-Claudie LARROCHE (1), Odile HOUCINE
}

\begin{abstract}
Département d'Anatomie normale et pathologique du Système nerveux central, Centre de Recherches de Biologie du Développement foetal et néonatal, INSERM U 29, 123, boulevard de Port-Royal, 75674 Paris Cedex 14.
\end{abstract}

Summary. Neocorticogenesis in human embryos and fetuses studied by electron microscopy and Golgi impregnations.

The telencephalic vesicle (or hemisphere) of human embryos and fetuses has been studied by electron microscopy and Golgi impregnations. Our observations confirm recent ones on various laboratory animals and revise some concepts of corticogenesis. At $4 / 6$ weeks, the neural tube was a single pseudostratified epithelium in which mitoses occurred. At 7 weeks the telencephalic vesicle was made up of two layers, the ventricular layer or matrix and the outer plexiform or marginal layer. The first cells, appearing in the plexiform layer before any cortical plate could be identified, had the morphological attributes of Cajal-Retzius cells. In this primitive layer we found the earliest synapses yet described in human embryos. From 8 weeks on, the cortical plate was formed by successive waves of cells, migrating from inside-out. The next synapses found were in the subcortical layer.

On Golgi impregnations the Cajal-Retzius cells were already visible at 15 weeks, and from 20 weeks on they presented the usual morphological characteristics.

\section{Introduction.}

Malgré la somme considérable de travaux sur les premières étapes du développement du système nerveux central des mammifères, quelques points restent encore obscurs ou controversés en ce qui concerne notamment la néocorticogenèse. Quant à l'embryon humain, les informations sont très rares, et même si l'on peut extrapoler à partir du chat ou de la souris, il n'en reste pas moins indispensable d'appliquer à son étude, à la fois les vieilles recettes d'imprégnations du type Golgi et les techniques plus modernes de microscopie électronique.

(1) Maître de Recherches au C.N.R.S. 


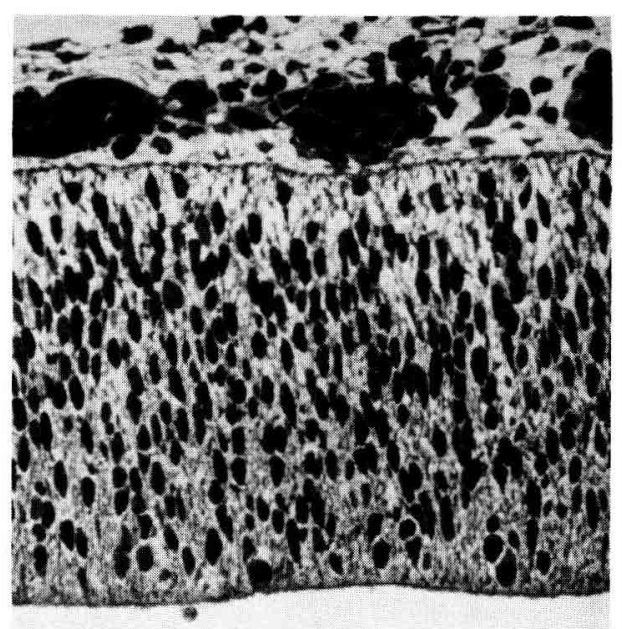

a

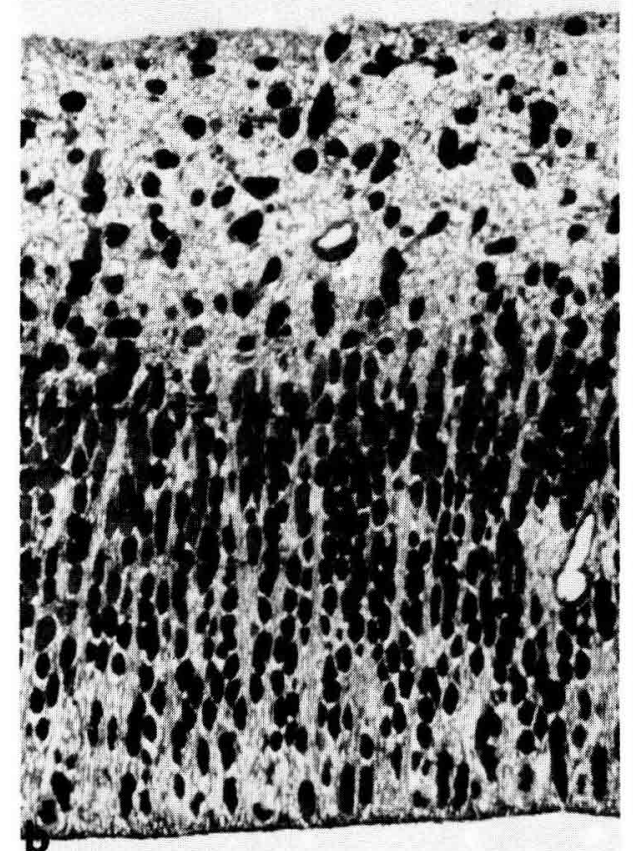

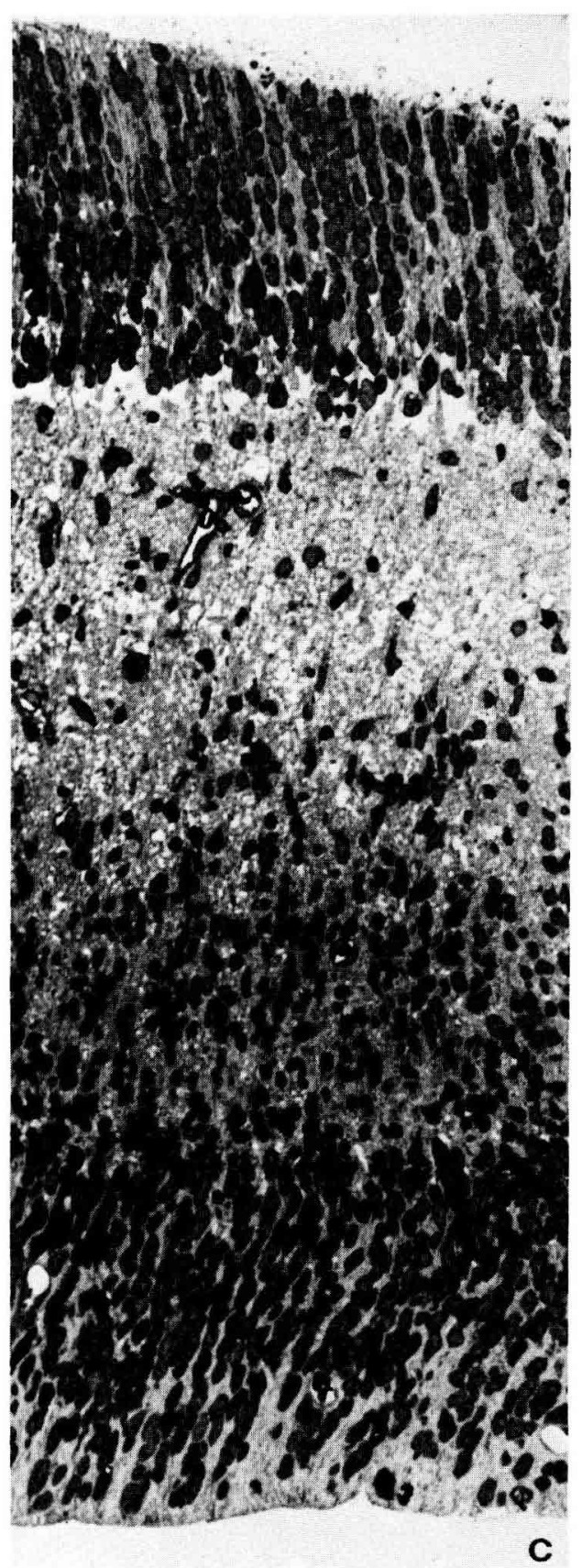

FIG. 1. - Evolution de la paroi de la vésicule prosencéphalique chez l'embryon humain: a) 6 semaines ; une couche pseudo stratifiée; b) $7 / 8$ semaines : matrix ou couche ventriculaire et couche marginale externe ; c) $9 / 10$ semaines : cinq couches : matrix ou couche ventriculaire, couche subventriculaire, couche intermédiaire, plaque corticale et couche moléculaire ou marginale externe. 


\section{Etat actuel de nos connaissances.}

Les toutes premières étapes de la neurogenèse ne sont certes plus discutées. Dès sa fermeture, le tube neural ébauche des courbures successives correspondant grossièrement dès la $4^{e}$ semaine aux différentes structures cérébrales, prosencéphale, mésencéphale et diencéphale, rhombencéphale et myélencéphale. La paroi, épithélium à l'origine unicellaire, est devenue chez l'embryon humain de 4 à 6 semaines, pseudo-stratifiée (fig. 1 a). De multiples couches de noyaux allongés, à chromatine dense peuvent être comptées. Il existe de nombreuses mitoses près de "l'épendyme " et l'on sait notamment depuis les travaux de Sauer (1959) que le noyau des cellules mères effectue un mouvement de va et vient dans la zone ventriculaire avant d'entrer en mitoses contre la paroi ventriculaire. Vers 78 semaines la vésicule télencéphalique est plus nette; le mésencéphale paraît énorme mais l'ébauche du cervelet est encore extrêmement petite. A cet âge la paroi de l'hémisphère a doublé d'épaisseur et comporte essentiellement deux couches : la couche interne ventriculaire ou matrix et la couche externe, plexiforme ou marginale (fig. 1 b). Deux semaines plus tard, les hémisphères commencent à recouvrir le mésencéphale ; il existe grossièrement 5 couches : la couche marginale, moléculaire ou couche I, la plaque corticale, la couche intermédiaire selon la terminologie classique de His, subdivisée en couches externe et interne, et la couche ventriculaire ou matrix (fig. $1 \mathrm{c}$ ).

$\mathrm{Au}$ cours de ces dernières décennies deux informations capitales nous ont été fournies. Premièrement grâce à l'utilisation de la thymidine tritiée (Angevine et Sidman, 1961) on a pu démontrer que les cellules filles issues de mitoses de la zone ventriculaire ou germinative, forment la plaque corticale de dedans en dehors ; les couches profondes sont ainsi traversées et " recouvertes " successivement par les couches les plus superficielles. Mais dans ce processus il n'est pas fait mention de la couche I, superficielle ou moléculaire.

La deuxième information concerne le mode de migration des neurones. En effet chez l'embryon humain et le fœetus humain, notamment au niveau du lobe occipital (Larroche, 1962) il est frappant de constater combien la migration cellulaire se fait, à partir de la couche ventriculaire, sur un mode radiaire perpendiculaire à la surface (fig. 2). Les mêmes observations faites chez le fœetus de singe ont été précisées par Rakic (1972) qui, grâce à des imprégnations de type Golgi, élabora un modèle selon lequel les cellules glio-épendymaires, tendues en pont de la paroi ventriculaire à la pie-mère serviraient de fil conducteur aux neurones. Le noyau de ces cellules se situe dans la zone ventriculaire et le pied se confond avec l'épithélium épendymaire (fig. 3). A la surface du cortex, leurs pieds, en étroit contact, reposent sur la membrane basale de la pie-mère et se détacheraient au cours du développement. Le prolongement apical, radiaire peut être suivi chez l'embryon humain dans la zone intermédiaire et dans la plaque corticale notamment, le long du dendrite apical du neurone contre lequel il s'applique.

Le point de nos connaissances sur les premières étapes du développement du système nerveux central, et la terminologie qu'il fut conseillé d'adopter ont été formulés en 1970 par le « Boulder Committee. " Chez l'embryon humain nous 
retrouvons bien le stade $A$ vers 4 à 6 semaines : c'est le cerveau à 1 couche (pseudo stratifié). Le stade B correspond à l'embryon humain de 7 à 8 semaines ou cerveau à 2 couches : couche marginale externe et couche germinative. Mais pour les auteurs du "Boulder Committee " la couche externe ou marginale " ne contient aucun type cellulaire qui lui soit propre "), assertion que nous croyons erronée. Au stade $\mathrm{C}$ apparaît, entre la couche marginale et la couche ventriculaire, la couche intermédiaire avec des cellules en voie de migration. Le stade D entre 8 et 9 semaines chez l'embryon humain (fig. 1 c) correspond au début de formation de la plaque corticale, sous la couche moléculaire ou marginale externe; les neurones traversent, pour ce faire, la couche intermédiaire. A ce stade, la couche ventriculaire n'est plus homogène et la partie la plus externe est appelée couche subventriculaire. En fait ces deux couches, intermédiaire et subventriculaire correspondent aux couches intermédiaires externe et interne décrites par His il y a près d'un siècle.

Enfin, le stade $E$ a 4 ou 5 couches selon que l'on subdivise ou non la couche subventriculaire. Chez l'adulte, la couche intermédiaire devient la substance blanche et la zone ventriculaire est réduite à l'épendyme.

Légèrement différente est la proportion de Marin Padilla (1978) qui envisage la formation du cortex des mammifères dans le cadre général de l'évolution phylogénétique où, chez l'amphibien par exemple, la substance blanche reste externe. Or la couche moléculaire qui est la première à s'individualiser très tôt est formée essentiellement par des fibres thalamo-corticales tangentielles à la surface. Cette couche est peuplée de grands neurones, les cellules de Cajal Retzius. La plaque corticale selon Marin Padilla va se former au sein même de cette couche plexiforme, la dédoublant en quelque sorte en couche I et couche VI profonde ou couche VII.

\section{Sujets d'étude et technique.}

La vésicule antérieure ou hémisphère, d'embryons et de fœtus humains âgés de 4 à 40 semaines, a pu être étudiée en microscopie photonique et ou électronique. Les techniques d'histologie traditionnelles ont été utilisées sur des fragments inclus en paraffine ou en celloïdine, sur des fragments fixés ou non pour les imprégnations de sels de métaux lourds (Golgi rapide et Golgi Cox) et enfin, lorsque les délais de prélèvements le permettaient, pour étude au microscope électronique (ME).

FIG. 2. - Paroi externe du lobe occipital chez un fœtus de 12/13 semaines. Noter l'aspect radiaire des cellules en voie de migration à partir de la couche ventriculaire.

FIG. 3. - Golgi rapide. Cellules glio-épendymaires chez un fœtus de 12 semaines.

FIG. 4. - Golgi rapide. Cellules de Cajal-Retzius chez deux fotus âgés respectivement de 15 et 25 semaines (dessins à la chambre claire). 

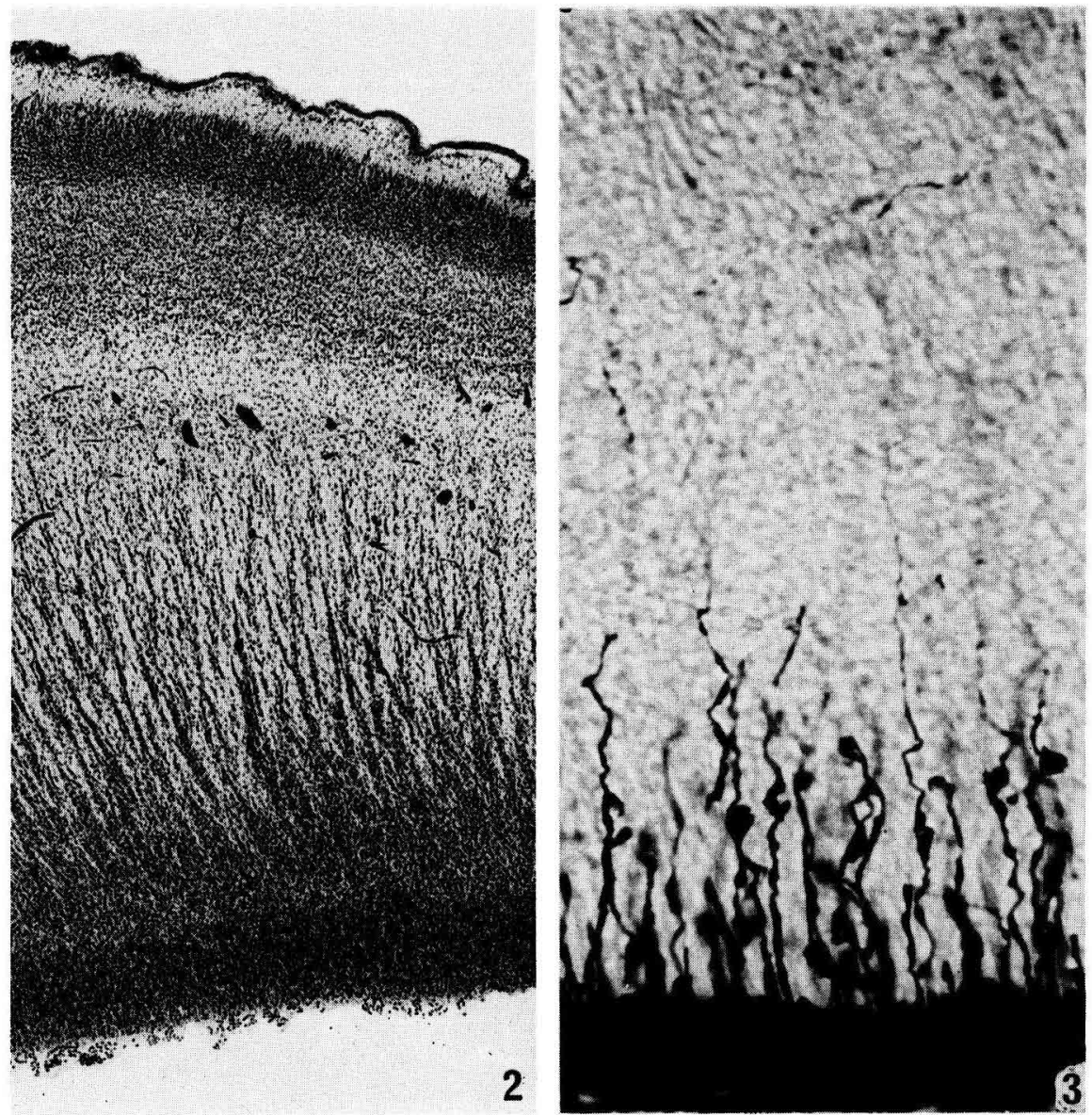

15

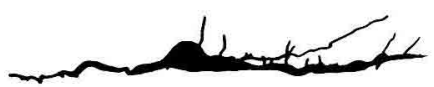

$4 a$

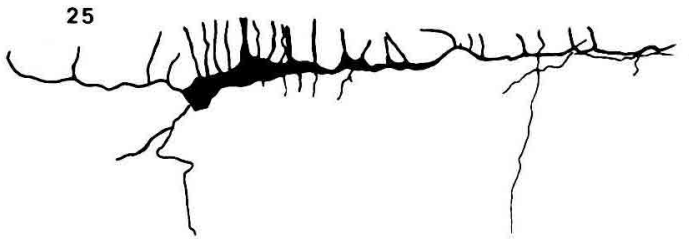

$4 b$ 
Ce travail porte sur la formation du néocortex et plus particulièrement sur la couche marginale externe, couche 1 sous-piale.

\section{Résultats et discussion.}

L'étude en microscopie photonique et électronique de la vésicule télencéphalique d'embryons humains de 4,7 et 10 semaines et les imprégnations de Golgi chez des fœetus de 15 à 40 semaines, nous ont permis de confirmer certains aspects du travail de Marin Padilla, travail effectué sur des embryons de chats.

- Premièrement, l'ancienneté de formation et la richesse de la couche moléculaire. Chez l'embryon humain de 7 semaines la vésicule télencéphalique présente déjà deux couches : la couche marginale externe qui est aussi épaisse que la couche germinative; elle a un aspect plexiforme lâche, fait d'axones et de fibres radiaires glio-épendymaires. L'espace extra-cellulaire est très grand. Il existe dans cette couche de nombreux grands neurones, les cellules de Cajal Retzius, dont l'axe est parallèle à la surface et dont le cytoplasme est extrêmement riche en organelles. Les techniques de Golgi sont difficiles et les résultats très aléatoires surtout chez l'embryon et le jeune fœtus. Cependant ces grandes cellules sont parfaitement reconnaissables dès la $15^{e}$ semaine (fig. 4 a). Au cours du développement, plusieurs types morphologiques de cellules de Cajal Retzius peuvent être reconnus selon l'orientation de leur grand axe et la répartition des branches collatérales (fig. $4 \mathrm{~b}, 5$ ). Très tôt différenciées puisque nous les avons observées en ME chez l'embryon de 7/8 semaines (fig. 6) on discute encore de leur rôle et de leur avenir. Leur nombre s'accroît-il encore après la formation de la plaque corticale ? Dans la négative, et si leur quota est définitif dès la $7 / 8^{\ominus}$ semaine, le peu de cellules de Cajal Retzius rencontrées chez l'enfant et l'adulte, voire leur absence pour certains ne serait alors dû qu'à une extrême " dilution " au cours du développement de la couche moléculaire. C'est également dans cette couche plexiforme ou marginale que nous avons trouvé les premières synapses (fig. 7) jamais décrites chez l'embryon humain de 7/8 semaines, témoin s'il en est de l'extrême élaboration des circuits neuronaux dès cet âge (Larroche, 1981).

- Notre deuxième observation concerne la couche sous-corticale ou couche VII de Marin Padilla. Chez le fœetus humain de 10 semaines, la plaque corticale est en place mais la région sous-corticale présente un aspect extrêmement lâche plexiforme rappelant la moléculaire ; et si les synapses sont retrouvées certes en grand nombre dans la couche moléculaire elles sont également nombreuses dans cette couche sous-corticale (fig. 8) et nulle part ailleurs (Larroche et al., 1981).

FIG. 5. - Golgi Cox. Cellules de Cajal-Retzius chez un nouveau-né à terme.

FIG. 6. - Cellules de Cajal-Retzius (ME $\times 2800$ ) chez un embryon de $7 / 8$ semaines.

FIG. 7. - Synapse dans la couche moléculaire; embryon de $7 / 8$ semaines. $\times 30000$.

FIG. 8. - Synapses sous la plaque corticale; fœtus de $9 / 10$ semaines. $\times 28000$. 


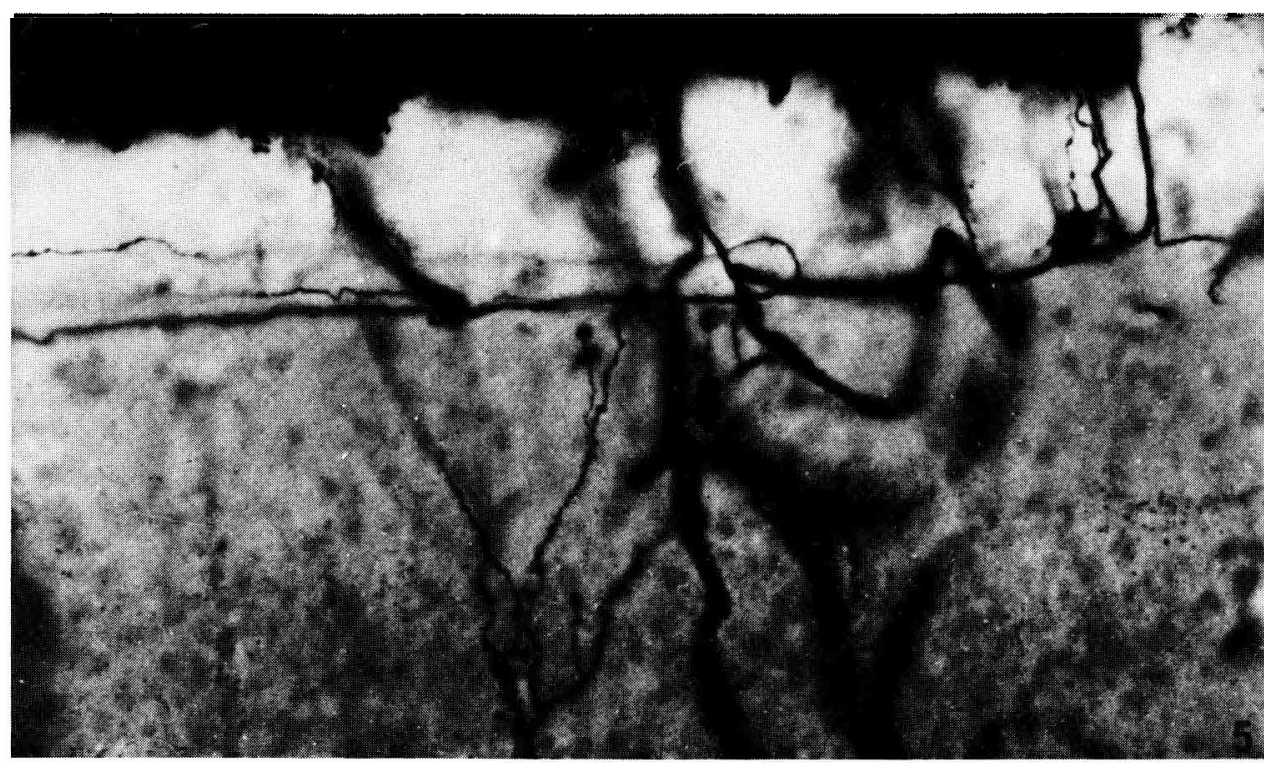

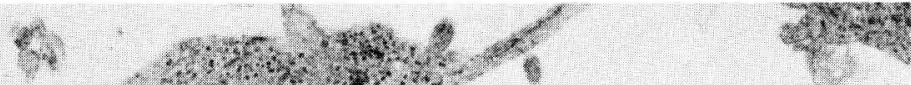

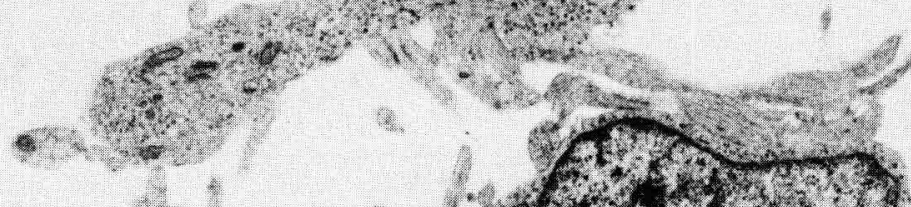

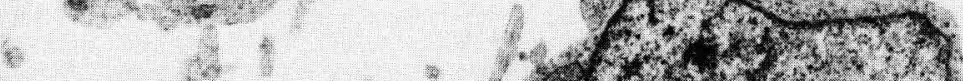
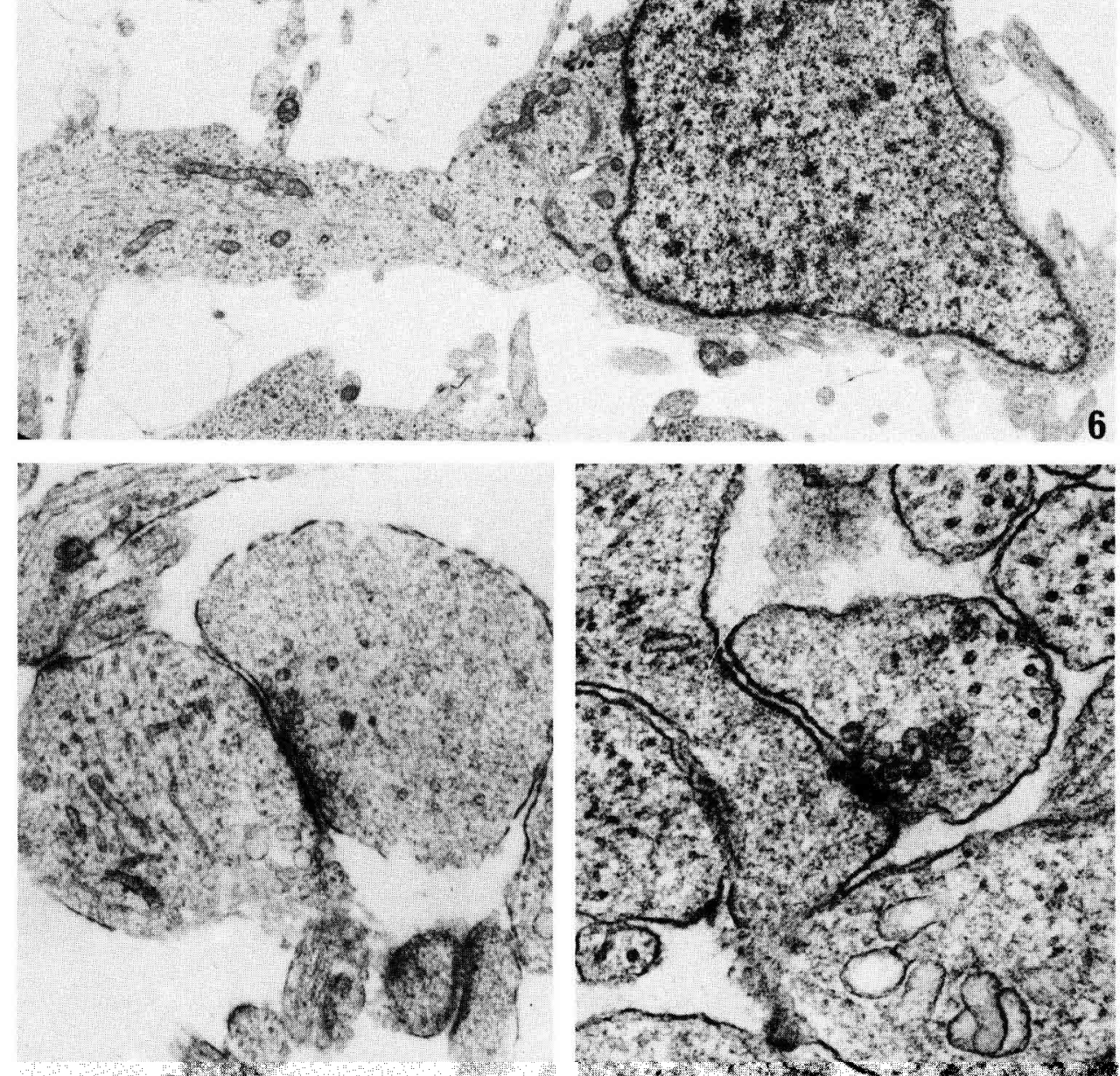

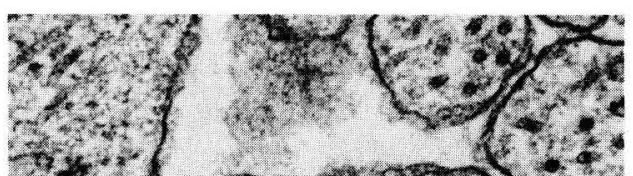

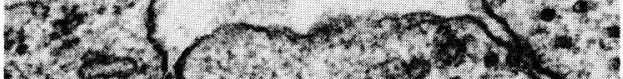

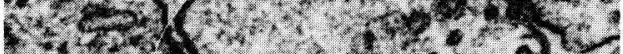

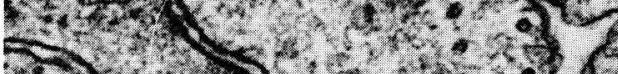

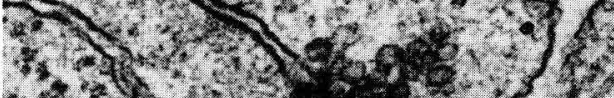

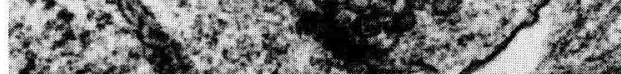

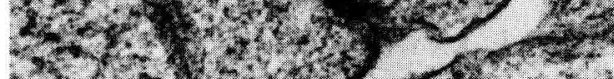

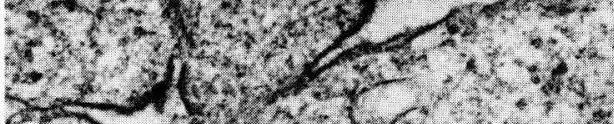

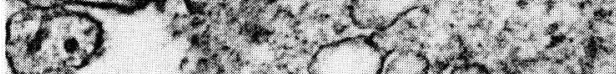

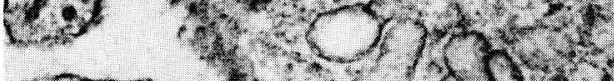
C:

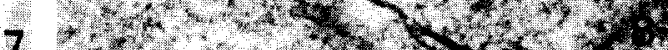


En conclusion, nous pouvons donc, à partir de l'examen du néocortex d'embryons et de fœtus humains, faire deux constatations :

1) II existe de grands neurones ou cellules de Cajal Retzius et des synapses dans la couche marginale externe avant même l'existence d'une plaque corticale, ce qui est en contradiction avec le concept général de l'élaboration corticale de dedans en dehors ou en serait une exception. Les cellules de Cajal Retzius présentent d'autre part, sur les imprégnations de Golgi, leurs caractères morphologiques classiques dès la $15^{\mathrm{e}}$ semaine.

2) L'observation chez l'embryon de $9 / 10$ semaines de synapses dans une zone lâche, d'aspect plexiforme, sous la plaque corticale, semble en faveur de la thèse de Marin Padilla faisant de cette couche (VII) un véritable dédoublement profond de la couche marginale primitive au sein de laquelle s'édifierait secondairement la plaque corticale.

7e Réunion du groupe Développement I.N.R.A., Nouzilly/Tours, 14-15 mai 1981.

Remerciements. - Nous remercions Madame Liliane Jardin pour son aide technique et le groupe de techniciens et photographes du laboratoire de Microscopie électronique du CNRS, bd Raspail, Paris.

\section{Références}

ANGEVINE J., SIDMAN R. L., 1961. Autoradiographic study of cell migration during histogenesis of cerebral cortex of the mouse. Nature (London), 192, 766-768.

BOULDER COMMITTEE, 1970. Embryonic vertebrate cerebral nervous system. Revised terminology. Anat. Rec., 166, 257-262.

LARROCHE J.-Cl., 1962. Quelques aspects anatomiques du développement cérébral. Biol. néonat., 4, 126-153.

LARROCHE J.-Cl., 1981. The marginal layer in the neocortex of a 7 week-old human embryo. Anat. Embryol., 162, 301-312.

LARROCHE J.-Cl., PRIVAT A., JARDIN L., 1981. Some fine structures of the human fetal brain, 350-358. In MINKOWSKI A. Physiological and biochemical basis for perinatal medicine. Sam. Levine int. Symp., Paris, 1979, Karger, Basel.

MARIN PADILLA M., 1978. Dual origin of the mammalian neocortex and evolution of the corticai plate. Anat. Embryol., 152, 109-126.

RAKIC P., 1972. Mode of cell migration to the superficial layers of the fetal monkey neocortex. J. comp. Neurol., 145, 61-84.

SAUER F. C., 1959. Radiographic study of the location of newly synthetized deoxyribonucleic acid in the neural tube of the chick embryo-evidence for intermitotic migration of nuclei. Anat. Rec., 133. 456. 